\title{
Plant polyphenols in prevention of heart disease
}

\author{
Ginter $E^{1}$, Simko $V^{2}$ \\ Institute of Preventive and Clinical Medicine, Bratislava, Slovakia. ginter.emil@mail.t-com.sk
}

\begin{abstract}
Polyphenols $(\mathrm{PPH})$ are a group of chemical substances found in plants, characterized by the presence of more than one phenol unit. The largest and best studied polyphenols are the flavonoids, which include several thousand compounds. PPH are the most abundant antioxidants in human diets. Their sheer mass in the diet exceeds that of the consumed vitamins. The Mediterranean diet is rich in PPH because it contains abundant vegetables, fruits, unrefined cereals, legumes, nuts, garlic, olive oil and red wine. Locations where the Mediteranean diet prevails are known for a reduced premature cardiovascular disease (CVD) mortality. However, this geographic distinction is not entirely unique. Low CVD mortality is also present in countries with high consumption if plant food and fish, as Scandinavia, Switzerland and Austria. Putative mechanisms of the metabolic effect of PPH are related to multiple biologic functions that may have beneficial effect in the prevention of some inflammation-mediated disorders, including CVD. Naturally occurring dietary PPH can directly scavenge free oxygen radicals, adhesion molecules, and growth factor receptor genes. Thus, these polyphenolic compounds have potential therapeutic value as antioxidant and anti-inflammatory agents in CVD. PPH reduce the generation of oxidized low density lipoproteins (LDL), induce nitric oxide (NO) production, inhibit platelet aggregation and downregulate expression of proinflammatory mediators (Fig. 6, Tab. 1, Ref. 33). Full Text in PDF www.elis.sk. Key words: polyphenols, flavonoids, resveratrol, curcumin, cardiovascular diseases.
\end{abstract}

Nutrition sciences in the first decades of the twentieth century became preoccupied with protective nutrients labelled as vitamins. These were attributed to organic compounds that the body is unable to synthetize, so they have to be provided in the food. Their insufficient supply leads to deficiency (hypo- or avitaminosis) that may result in disease or even death. Such vitamin concept does not include other essential nutrients such as minerals, essential amino acids and fatty acids, nor does it encompass the large number of other nutrients that promote health.

Polyphenols (PPH) rank among nutrients having an important potential for maintaining health. The largest and best studied PPH are the flavonoids, which include several thousand compounds, among them the flavonols, flavones, catechins, flavanones, anthocyanidins, and isoflavonoids. Nutritional deficiency of flavonoids is not equivalent to ,avitaminosis“ although it is associated with a negative impact on health.

Dietary flavonoids represent a wide variety of compounds that occur in vegetables, fruits, legumes, nuts, cereals, wine, tea, virgin olive oil and cocoa products. Presently there are over 8,000 structural variants of flavonoids, bearing aromatic rings and one or more hydroxyl moieties. In the past few years flavonoids have been very much in biomedical focus. PubMed refers to over 52,000 papers dealing with the subject of flavonoids.

${ }^{1}$ Institute of Preventive and Clinical Medicine, Bratislava, Slovakia, and ${ }^{2}$ State University of New York, Downstate Medical Center at Brooklyn, USA

Address for correspondence: E. Ginter, RND, DSc, Racianska 17, SK83102 Bratislava, Slovakia.
The reader interested in more details on the subclasification and chemical structure of PPH may find ample additional information $(1,2)$. The aim of this review is to bring attention only to PPH that have the most promising potential in assisting with prevention of cardiovascular disorders.

\section{Polyphenols and cardiovascular disease}

Phenols are a class of chemical compounds consisting of a hydroxyl group (-OH) bonded directly to an aromatic hydrocarbon group. Phenol forms a redox system with chinone (Fig. 1) and therefore dietary PPH can directly scavenge free oxygen radicals and downregulate expression of proinflammatory mediators, adhesion molecules, and growth factor receptor genes. Thus, these polyphenolic compounds have potential therapeutic value as antioxidant and anti-inflammatory agents in CVD.

\section{Oxidation of phenol to chinone}

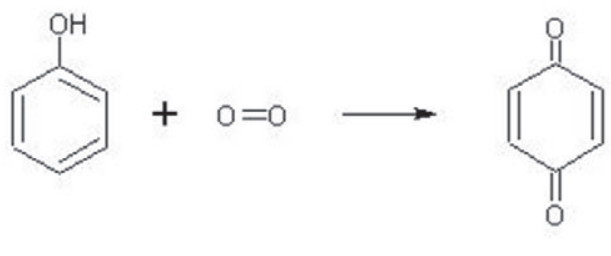

Phenol

Chinone

Fig. 1. Redox system of phenol and chinone. 
Potential evidence for health benefit of dietary PPH is the relatively low CVD mortality in geographic regions where the population consumes a diet high in fruit, vegetables and other plant food (3). In addition to direct effect of PPH on metabolic processes leading to lipid deposition in the arterial wall, there are other, until recently little explored venues where PPH may decrease predisposition to CVD. Low CVD in southern Europe, in France, Italy and Spain may also be related to other factors of the life style, especially the emotional well being (4). Recent years have brought solid scientific evidence for a direct association between severe depression and the atherogenesis $(5,6)$. Cerebral blood flow must be maintained to ensure a constant delivery of oxygen and glucose as well as the removal of waste products. The prospect for increasing blood flow with dietary polyphenols is extremely promising (7). The high demand of brain for molecular oxygen, high level of polyunsaturated fatty acids in membrane phospholipids and low activity of antioxidant defense enzymes are factors rendering cells in the central nervous system particularly vulnerable to oxidative stress.

This review by no means pretends to analyze the effect of all PPH on the CV system: such account would be too wide. The focus is only on the most relevant PPH that have been definitely confirmed to bear a favorable effect on metabolic pathways which link to the CV system.

\section{Resveratrol}

Although resveratrol was first isolated in 1940, the importance of resveratrol was recognized only after the widely publicized<smiles>[R6][R3][Ca]</smiles>

Fig. 2. Chemical structure of resveratrol.
"French Paradox" associated with drinking of red wine. Resveratrol (3,5,4'-trihydroxystilbene) is a polyphenolic phytoalexin (Fig. 2 ). It is produced in the skin of grapes by the effect of fungi and bacteria. Resveratrol is also present in many other plant products. The highest concentrations of resveratrol are found in red Spanish wines (over $12 \mathrm{mg} / \mathrm{L}$ ). Experimental studies have provided evidence that resveratrol possesses antioxidant (oxidative modification of LDL), antiapoptotic, angiogenic, antihypercholesterolemic, and antidiabetic effects. Exposure to resveratrol stimulates nitric oxid (NO) production, protects endothelial cells from oxidative functional damage, also affects the antiplatelet activity and mononucleocyte adhesion $(8,9,10,11)$.

In humans, resveratrol, at the concentrations produced in plasma by moderate intake of wine, stimulated in platelets NO production and lowered platelet aggregation (Fig. 3) (12). Resveratrol may hold promise in the management of myocardial ischemia and ox-LDL-hypercholesterolemia. Resveratrol seems to be well tolerated and no marked toxicity was reported. These data are important in the context of human efficacy studies, and they provide further support for the use of resveratrol as a pharmacological drug in human medicine $(12,13)$. Resveratrol likely fulfills the definition of a pharmacological preconditioning compound and gives hope for the therapeutic promise of alternative medicine (14).

\section{Enterolactone}

This interesting PPH differs from other PPH in that it is not derived directly from food but that it is generated in the colon by

\section{Enterolactone}

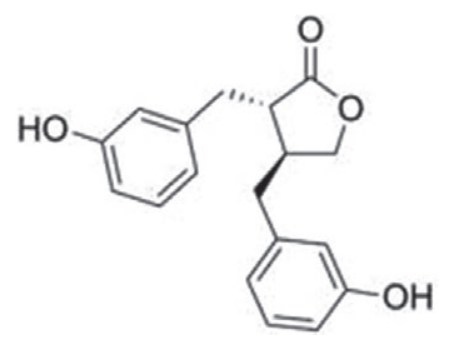

Fig. 4. Chemical structure of enterolactone.

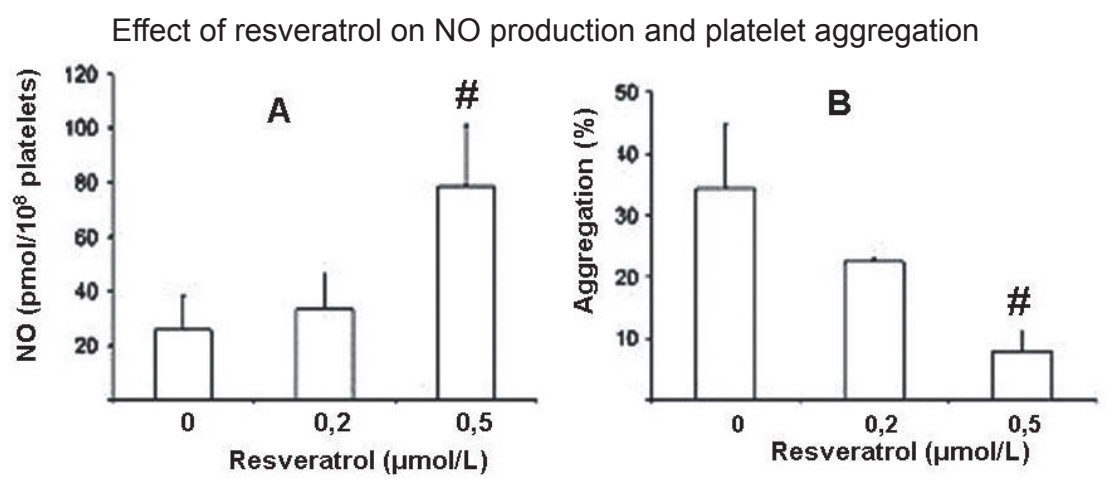

Fig. 3. Effect of resveratrol on collagen induced NO production and platelet aggregation in humans. (A) NO release from collagen-stimulated platelets. (B) Maximal amplitude of aggregation of collagen-stimulated washed platelets. According to Gresele et al (12). 


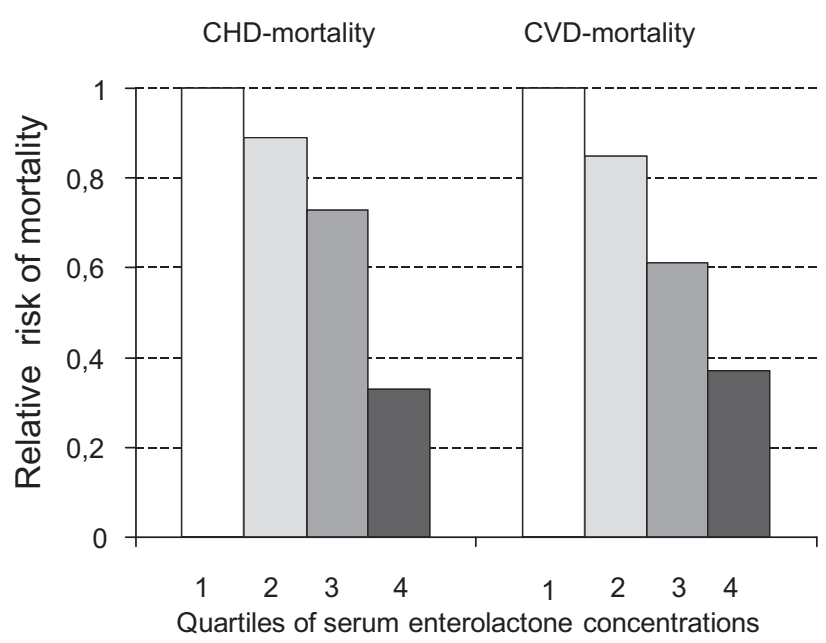

Fig. 5. The relative risks for coronary heart disease and cardiovascular disease mortality by quartiles of serum enterolactone concentration. Quartile 1 indicates 0.2-6.9 nmol/L, quartile 2: 7.0-13.7 nmol/L, quartile $3: 13.8-23.8 \mathrm{nmol} / \mathrm{L}$ and quartile $4: 23.9-88.7 \mathrm{nmol} / \mathrm{L}$. According to Vanharanta et al.(15).

the activity of intestinal microorganisms which affect plant lignans. Resulting enterolactone is not only an important biomarker of dietary intake of vegetables and dietary fiber but its phenolic structure is also an important antioxidant (Fig. 4). There are reports associating high levels of enterolactone with reduced risk of coronary heart disease and cardiovascular disease mortality (Fig. 5) (15). High intake of fresh fruit, root vegetables, cereals and fruiting vegetables was associated with reduced mortality of coronary heart disease and stroke in a prospective study on more than 40,000 males and females in Spain and in many other countries (16).

\section{Curcumin}

Curcumin is a yellow-colored polyphenolic pigment obtained from the rhizome of Curcuma longa Linn. The root of turmeric has long been used in traditional Asian medicine. Animal experiments demonstrated anti-inflammatory, antioxidant, and anti-cancer properties of curcumin (17). In animal models curcumin ameliorates the development of cardiac hypertrophy and heart failure (18). Curcumin was found to be much faster in terms of quenching free oxygen radicals when compared to resveratrol or quercetin. Curcumin has been demonstrated to induce antioxidant defenses through increase in glutathione production. The anti-thrombotic, anti-proliferative, and anti-inflammatory effects of curcumin and the effect of curcumin in decreasing the LDL-cholesterol level may protect against the pathological changes occurring with atherosclerosis.

Initially it was postulated that curcumin cannot be absorbed from the human gastrointestinal tract. More recently it was reported that curcumin is absorbed after oral dosing in humans and can be detected as glucuronide and sulfate conjugates in plasma (19). Preliminary human evidence suggests possible efficacy of curcumin in the management of heart disease (20). Further clinical trials are needed to fully develop the potential of curcumin.

\section{Sources of polyphenols - problem of dietary supplements}

Intensive interest in PPH dates back more than ten years. This has also been reflected in a remarkable commercial response of the dietary supplement industry, of the alternative medicine establishment, along with vigorous marketing. Dietary supplements are based on the assumption that dietary sources of essential nutrients do not provide adequate protection and protective substances have to be administered in a non-dietary form.

Such a suggestion is questionable. Individuals consuming adequate amount of plant diet, receive in their diet large and variable sources of PPH.

Fruits such as grapes, pears, apples, plums, raspberries, blackberries, blueberries, cantaloupe, cherries, cranberries, pears and strawberries, most legumes and vegetables such as cabbage, celery, onion, broccoli and parsley are rich in PPH. Many grains, red wine, chocolate, green tea and olive oil are also rich sources of PPH. This is why the role of dietary supplements as a method of realizing health benefits of PPH is the subject of considerable discussion.

Enormous amount of money is spent on products that have not been approved by the controlling agencies that decide on the basis of scientific evidence (e.g. FDA, Food and Drug Administration in the USA). Remarkably, after more than ten years there has been no decisive ruling on the health efficacy, safe dose and absence of side effects of the PPH. Because of uncertainty about the metabolic efficiency of $\mathrm{PPH}$, a dietary reference intake for PPH has not been established (21). To obtain scientific validity of health benefits of PPH, adequate data on the bioavailability, dose effect and toxic levels have to be established. Structural difference in the large number of PPH makes it difficult to study their biological effect and to set daily recommended doses for at least the most important PPH.

Resveratrol, one of the most prominent PPH is a good example.

Words of caution include its potential side effects: there is a mild hormonal effect, estrogenic and anti-growth (the product should not be used in small children). Resveratrol reduces the activity of some enzymes involved in the metabolism of medications. Because of its potential anti-platelet effect, it has to be used with caution in patients who take blood thinners.

\section{Conclusion}

The large group of plant PPH includes more than ten subclasses. What concerns the antioxidant capacity of human diet, the contribution of PPH is much larger than that of the vitamins. Flavonoids, the water-soluble plant pigments are the most important group of PPH and their total intake could amount to almost $1 \mathrm{~g} / \mathrm{d}$, much higher than the intake of all dietary vitamins with antioxidative effect (21) (Tab. 1). This review confirms the accumulating evidence that PPH possess a diverse array of biological actions and may be beneficial in the prevention of some inflammatory-mediated diseases including cardiovascular disease. Unfortunately, at this time, no randomized controlled trial studied effects of PPH on CVD.

However, meta analyses of prospective studies indicate that high dietary intake of flavonols may be associated with a reduced 
Tab. 1. Intake as polyphenols in mg per $100 \mathrm{~g}$ of selected foods according to the USDA database. According to Williamson and Holst (21).

\begin{tabular}{lcccc}
\hline & Flavanones & Flavonols & Flavanols & Procyanidins \\
\hline Orange & 44 & 0 & 0 & 0 \\
Red onion & 0 & 39 & 0 & 0 \\
Blueberries & 0 & 4 & 3 & 325 \\
Strawberry & 0 & 2 & 4 & 13 \\
Apple & 0 & 4 & 9 & 113 \\
Total & 44 & 49 & 16 & 128 \\
\hline
\end{tabular}

\section{Bioactivities of plant polyphenols}

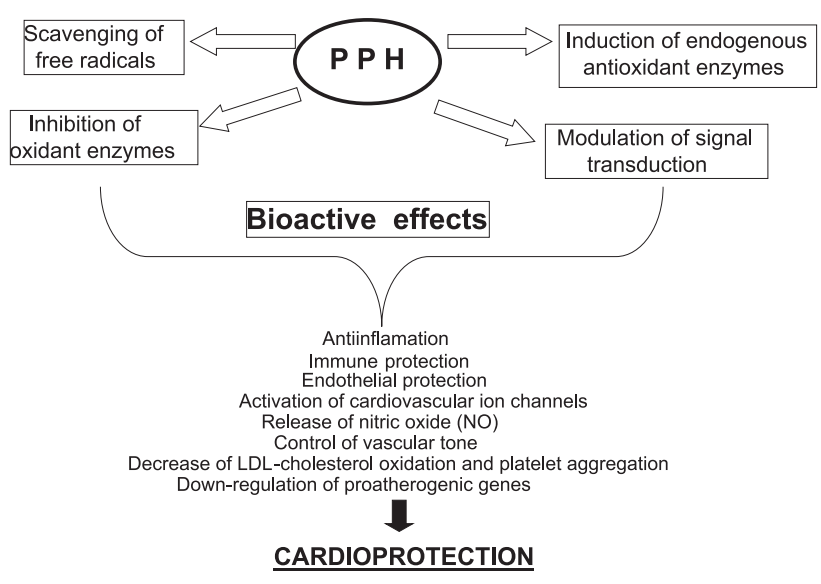

Fig. 6. Schematic effects of plant polyphenols bioactivities and their effects in cardioprotection.

risk from CVD in free-living populations (22-26). A number of trials of acceptable quality with flavonoid-rich foods provide evidence for a beneficial effect on flow-mediated dilation, blood pressure and lipid profile, which are relevant indicators of cardiovascular health.

Optimal doses of specific flavonoids for CVD protection are not known, but there are new data from 2010 on the protective effects of berries (chokeberries, cranberries, blueberries, and strawberries) such as upregulation of endothelial nitric oxide synthase, decreased activities of carbohydrate digestive enzymes, decreased oxidative stress, and inhibition of inflammatory gene expression and foam cell formation (27). Benefits were seen in healthy subjects and in those with existing metabolic risk factors. The results with pomegranate juice are promisimg: this juice significantly reduced atherosclerotic lesion areas in immune-deficient mice and intima media thickness in cardiac patients. It also decreased lipid peroxidation in patients with type 2 diabetes, and systolic blood pressure and serum angiotensin converting enzyme activity in hypertensive patients (28). There is an increasing body of evidence that flavonoids specifically target molecular structures including cardiovascular ion channels (29).

Red wine polyphenols are able to activate extracellular regulated kinase and p38 kinase and switch off the NF-kappaB pathway via an increased expression with time of the IkappaBalpha (IкB-alfa) phosphorylated form (30). These mechanisms may represent key molecular events leading to inhibition of the proinflammatory cascade and atherogenesis. PPH have the capacity to improve the endothelial control of vascular tone not only in several experimental models of cardiovascular diseases such as hypertension but also in healthy and diseased humans (31). The experiments on healthy people indicate a significant role of olive oil polyphenols in the down-regulation of proatherogenic genes. The benefits associated with olive oil polyphenol consumption on cardiovascular risk can be mediated through nutrigenomic effects (32). Figure 6 summarizes the bioactivities of PPH with the resulting cardioprotective effects.

The supplementation of cardiovascular friendly natural products needs to be considered in all populations who have high prevalence of CVD. Although PPH-rich foods are likely to protect cardiovascular system, long-term clinical trials are needed to definitively clarify the benefits deriving from long-term consumption of PPH-rich foods, particularly focusing on the lowest effective levels as well as synergism or antagonistic actions between different classes of PPH (33).

\section{References}

1. Han X, Shen T, Lou H. Dietary polyphenols and their biological significance. Int J Mol Sci 2007; 8: 950-988.

2. Neveu V, Perez-Jiménez J, Vos $\mathbf{F}$ et al. Phenol-Explorer: An online comprehensive database on polyphenol contents in foods. Database 2010; available at http://www.phenol-explorer.eu)

3. European Health for All Database, WHO Regional Office for Europe, Copenhagen, updated July 2010.

4. Sánchez-Villegas A, Delgado-Rodríguez M, Alonso A et al. Association of the Mediterranean dietary pattern with the incidence of depression. Psychiatry, 2009; 66: 1090-1098.

5. Sher Y, Lolak S, Maldonado JR. The impact of depression in heart disease Curr Psychiatry Rep 2010; 12 (3): 255-264.

6. Khan FM, Kulaksizoglu B, Cilingiroglu M. Depression and coronary heart disease. Curr Atheroscler Rep 2010; 12 (2):105-109.

7. Ghosh D, Scheepens A. Vascular action of polyphenols. Mol Nutr Food Res 2009; 53 (3): 322-331.

8. Penumathsa SV, Maulik N. Resveratrol: a promising agent in promoting cardioprotection against coronary heart disease. Can J Physiol Pharmacol 2009; 87: 275-286.

9. Chow SE, Hshu YC, Wang JS et al. Resveratrol attenuates oxLDLstimulated NADPH oxidase activity and protects endothelial cells from oxidative functional damages. Appl Physiol 2007; 102: 1520-1527.

10. Zhang $\mathbf{H}$; Zhang J, Ungvari $Z$ et al. Resveratrol improves endothelial function. Arterioscl Thromb Vascr Biol 2009; 29: 1164-1171. 
476-480

11. Schini-Kerth VB, Auger C, Kim JH et al. Nutritional improvement of the endothelial control of vascular tone by polyphenols: role of $\mathrm{NO}$ and EDHF. Pflugers Arch 2010; 459 (6): 853-62.

12. Gresele P, Pignatelli P, Guglielmini G et al. Resveratrol, at concentrations attainable with moderate wine consumption, stimulates human platelet nitric oxide production. J Nutr 2008; 138: 1602-1608.

13. Cottart CH, Nivet-Antoine V, Laguillier-Morizot C, Beaudeux JL. Resveratrol bioavailability and toxicity in humans. Mol Nutr Food Res 2010; 54 (1): 7-16.

14. Das S, Das DK. Resveratrol: a therapeutic promise for cardiovascular diseases. Recent Pat Cardiovasc Drug Discov 2007; 2 (2): 133-138.

15. Vanharanta M, Voutilainen S, Rissanen TH et al. Risk of cardiovascular disease-related and all-cause death according to serum concentrations of enterolactone: Kuopio Ischaemic Heart Disease Risk Factor Study. Arch Intern Med 2003; 163: 1099-1104.

16. Agudo A, Cabrera L, Amiano $P$ et al. Fruit and vegetable intakes, dietary antioxidant nutrients, and total mortality in Spanish adults: findings from the Spanish cohort of the European Prospective Investigation into Cancer and Nutrition ( EPIC-Spain). Amer J Clin Nutr 2007; 85: 1634-1642.

17. Rahman I. Dietary polyphenols mediated regulation of oxidative stress and chromatin remodeling in inflammation. Nutr Rev 2008; 66: S42-S45.

18. Wongcharoen W, Phrommintikul A. The protective role of curcumin in cardiovascular diseases. Int J Cardiol 2009; 133: 145-151.

19. Vareed SK, Kakarala M, Ruffin MT et al. Pharmacokinetics of curcumin conjugate metabolites in healthy human subjects. Cancer Epidemiol Biomarkers Prev 2008; 17: 1411-1417.

20. Aggarwal BB, Harikumar KB. Potential therapeutic effects of curcumin, the anti-inflammatory agent, against neurodegenerative, cardiovascular, pulmonary, metabolic, autoimmune and neoplastic diseases. Int J Biochem 2009; 41: 40-59.

21. Williamson G, Holst B. Dietary reference intake ( DRI) for dietary polyphenols: are we heading in the right direction? Brit J Nutr 2008; 99 (Suppl 3): S 55-S58.
22. Hooper L, Kroon PA, Rimm EB et al. Flavonoids, flavonoid-rich foods, and cardiovascular risk: a meta-analysis of randomized controlled trials. Am J Clin Nutr 2008; 88: 38-50.

23. Geleijnse JM, Hollman PCh. Flavonoids and cardiovascular health: which compounds, what mechanisms? Am J Clin Nutr 2008; 88:12-13.

24. Huxley RR, Neil HA. The relation between dietary flavonol intake and coronary heart disease mortality: a meta-analysis of prospective cohort studies. Eur J Clin Nutr 2003; 57: 904-908.

25. Hollman P, Geelen A, Kromhout D. Dietary flavonol intake may lower stroke risk in men and women. J Nutr 2010; 140: 600-604.

26. Panagiotakos DB, Dimakopoulou K, Katsouyanni K et al. Mediterranean diet and inflammatory response in myocardial infarction survivors. Int. J. Epidemiol 2009; 38: 856-866.

27. Basu A, Rhone M, Lyons TJ. Berries: emerging impact on cardiovascular health. Nutr Rev 2010; 68 (3): 168-177.

28. Basu A, Penugonda K. Pomegranate juice: a heart-healthy fruit juice. Nutr Rev 2009; 67 ( 1): 49-56.

29. Scholz EP, Zitron E, Katus HA et al. Cardiovascular ion channels as a molecular target of flavonoids. Cardiovasc Ther 2010; 28 (4): e46-52.

30. Magrone T, Jirillo E. Polyphenols from red wine are potent modulators of innate and adaptive immune responsiveness. Proc Nutr Soc 2010; 69 (3): 279-285.

31. Schini-Kerth VB, Auger C, Kim JH et al. Nutritional improvement of the endothelial control of vascular tone by polyphenols: role of $\mathrm{NO}$ and EDHF. Pflugers Arch 2010; 459 (6): 853-62.

32. Konstantinidou V, Covas MI, Muñoz-Aguayo D et al. In vivo nutrigenomic effects of virgin olive oil polyphenols within the frame of the Mediterranean diet: a randomized controlled trial. FASEB J 2010; 24 (7): 2546-2557.

33. Grassi D, Desideri G, Croce $\mathbf{G}$ et al. Flavonoids, vascular function and cardiovascular protection. Curr Pharm Des 2009; 15 (10): 1072-1084.

Received November 22, 2010. Accepted April 15, 2012. 